\title{
Intensification of heat-transfer and mixing in multifunctional heat exchangers by artificially generated streamwise vorticity
}

\author{
S. Ferrouillat ${ }^{\text {a }}$, P. Tochon ${ }^{\text {a }}$, C. Garnier ${ }^{\text {a }}$, H. Peerhossaini ${ }^{\text {b,* }}$ \\ ${ }^{\text {a }}$ Commissariat of Atomic Energy-GRETh, 17 avenue des Martyrs, F-38054 Grenoble Cedex 9, France \\ b Thermofluids, Complex Flows and Energy Group, Laboratoire de Thermocinétique, CNRS-UMR 6607, Ecole Polytechnique de l'Université de Nantes, \\ Rue Christian Pauc, BP 50609, F-44306 Nantes Cedex 3, France
}

Compact heat exchangers are well known for their ability to transfer a large amount of heat while retaining low volume and weight. The purpose of this paper is to study the potential of using this device as a mixer as well as a chemical reactor, generally called a multifunctional heat exchanger (MHE). Indeed, the question arises: can these geometries combine heat transfer and mixing in the same device? Such a technology would offer many potential advantages, such as better reaction control (through the thermal aspect [S. Ferrouillat, P. Tochon, H. Peerhossaini, D. Della Valle, Open-loop thermal control of exothermal chemical reactions in multifunctional heat exchangers, Int. J. Heat Mass Transfer, in press]), improved selectivity (through intensified mixing, more isothermal operation and shorter residence time, and sharper residence time distribution (RTD)), byproduct reduction, and enhanced safety.

Several geometries of compact heat exchanger based on turbulence generation are available. This paper focuses on one type: vortex generators. The main objective is to contribute to the determination of turbulent flow inside various geometries by computational fluid dynamics methods. These enhanced industrial geometries are studied in terms of their thermal-hydraulic performance and macro-/micromixing ability [S. Ferrouillat, P. Tochon, H. Peerhossaini, Micromixing enhancement by turbulence: application to multifunctional heat exchangers, Chem. Eng. Process., in press]. The longitudinal vortices they generate in a channel flow turn the flow perpendicular to the main flow direction and enhance mixing between the fluid close to the fin and that in the middle of the channel. Two kinds of vortex generators are considered: a delta winglet pair and a rectangular winglet pair. For both, good agreement is obtained between numerical results and data in the literature. The vortex generator concept is found to be very efficient in terms of heat-transfer enhancement and macro-mixing. Nevertheless, the micro-mixing level is poor due to strong inhomogeneities: the vortex generator must be used as a heattransfer enhancement device or as a static mixer for macro- and meso-mixing.

Keywords: Multifunctional heat exchanger; Vortex generator; Streamwise vortex; Intensification; Mixing; Heat-transfer enhancement; Large eddy simulation

\section{Introduction}

Compact plate-fin heat exchangers were initially developed in the 40s in the aerospace industry to provide compact, light, highly efficient heat exchangers for gas/gas

\footnotetext{
* Corresponding author. Tel.: +33 (0)2 406831 42; fax: +33 (0)2 4068 3141.

E-mail address: hassan.peerhossaini@univ-nantes.fr (H. Peerhossaini).
}

applications. These heat exchangers can provide secondary surfaces up to $90 \%$ of the overall heat transfer surface that lead to high heat transfer area per unit volume (usually above $700 \mathrm{~m}^{2} / \mathrm{m}^{3}$ ). Several types of geometries are available, the selection among which depends essentially on the application. In the chemical processing industry, the heat exchanger has a potential application as a reactor. Indeed, the high mixing level and the high heat-transfer performance of turbulence promoters (such as vortex generators) contained in a compact heat exchanger makes this 


\section{Nomenclature}

$$
\begin{array}{ll}
A_{\mathrm{F}} & \text { fin area, } \mathrm{m}^{2} \\
A_{\mathrm{VG}} & \text { VG area, } \mathrm{m}^{2} \\
B & \text { channel width, } \mathrm{m} \\
C f & \text { drag coefficient, dimensionless } \\
C p & \text { pressure coefficient, dimensionless } \\
e & \text { fractional liquid hold-up, dimensionless } \\
E_{\mathrm{D}} & \text { direct dissipation, } \mathrm{m}^{2} \mathrm{~s}^{-3} \\
d_{\mathrm{h}} & \text { hydraulic diameter, } \mathrm{m} \\
f & \text { fanning friction factor, dimensionless } \\
H & \text { channel height, } \mathrm{m} \\
j & \text { Colburn factor, dimensionless } \\
l & \text { VG span, m } \\
L & \text { channel length, } \mathrm{m} \\
N u & \text { Nusselt number, dimensionless } \\
Q & \text { volumetric flow rate, } \mathrm{m}^{3} \mathrm{~s}^{-1} \\
U & \text { flow velocity, } \mathrm{m} \mathrm{s}
\end{array}
$$

$\begin{array}{ll}V_{\mathrm{M}} & \text { working volume, } \mathrm{m}^{3} \\ s & \text { distance between tips of winglet pair, } \mathrm{m} \\ x_{v} & \text { distance of wingtips from the channel entrance, } \\ & \mathrm{m} \\ z & \text { VG height, } \mathrm{m} \\ \text { Greek } & \text { letters } \\ \beta & \text { angle of attack, } \\ \Delta P & \text { pressure drop, Pa } \\ \varepsilon & \text { turbulent energy dissipation, } \mathrm{m}^{2} \mathrm{~s}^{-3} \\ \eta & \text { mixing efficiency, dimensionless } \\ \mu & \text { dynamic viscosity, Pa s } \\ v & \text { kinematic viscosity, } \mathrm{m}^{2} \mathrm{~s}^{-1} \\ \rho & \text { fluid density, kg } \mathrm{m}^{-3} \\ \Phi & \text { average rate of energy dissipation, } \mathrm{m}^{2} \mathrm{~s}^{-3}\end{array}$

device potentially useful as a chemical reactor. This technology offers many advantages such as better reaction control through the thermal control of the reactor $[1,2]$, improved selectivity (through intensified mixing, more isothermal operation and shorter residence time, and sharper RTD), byproduct reduction and better safety. Indeed, although many traditional designs such as stirred tanks already incorporate heat transfer, in these configurations there is a significant distance between the heat transfer device and the site of the chemical reaction. The aim of the multifunctional heat exchanger (MHE) is to reduce this distance by supplying or removing the heat almost as rapidly as it is absorbed or generated by the reaction $[3,4]$.

In the last three decades, two different approaches have been used to enhance heat transfer in heat exchangers. One approach has focused on the increase of heat transfer area per unit volume and/or the increase of convective heat transfer coefficient through the reduction of the channel hydraulic diameter. Usually, this reduction implies to work in laminar flow. The second approach consists in working in turbulent regime and the extension of the heat transfer area is also achieved by using fins or grooves. The increase of the convective heat transfer coefficient is attained by mixing the main flow with the flow in the wall region, reducing the flow boundary layer thickness and raising the turbulence intensity. Unfortunately, this approach in turbulent flow involves a dramatic increase of the pressure drops.

In this paper, we focus on transitional flow $(2000<$ $R e<8000)$. An interesting compromise to reach high convective heat transfer coefficients with reasonable pressure drops is to use specifics geometries which generate longitudinal vertical structures.

The development of such devices requires sizing tools to aid in the design and operation of the process. The thermal performance of heat exchangers is key to a global energy efficiency analysis. Furthermore, local analysis of flow and heat transfer conditions is also required for better characterization in terms of chemical reactors (mixing intensity and residence time distribution).

It has been shown that longitudinal vertical structures improve considerably heat and mass transfer between a solid wall and a fluid layer [5]. This heat-transfer enhancement is driven by the velocity field. Incompressibility conditions require that the flow field and temperature field be uncoupled. Therefore, heat is transported, as a passive quantity, by the flow field. The principal aspects of the heat-transfer problem can thus be captured from the corresponding hydrodynamic problem.

Longitudinal vertical structures can have a variety of different forms according to the manner in which they are generated. They fall into two main categories.

- Vortical structures generated directly due to a mechanism inherent to the flow, such as centrifugal instability, cross-flow instability, etc. A common example of this category is the Görtler vortex [6-8].

- Those generated artificially or indirectly in a flow and then carried or embedded in the boundary layer. Examples of the second category are delta-wing vortices $[9,10]$, vortices generated by roll-up of fluid on the corners of the junction of a bluff body with a flat plate or behind a wire grid.

The main difference between the two categories is the driving force for generation of the vortices. In the case of Görtler vortices, for example, the driving mechanism is the centrifugal instability. As long as the instability conditions are satisfied, vortices can maintain their motion. This is not the case for those in the second category since, once the vortical structure is generated in the flow, no energy is 
input to maintain vortex strength. Therefore, the vortices break-down into turbulence (turbulence promoters) and decay downstream due to the viscous dissipation.

In this paper we focus on a technology (vortex generator) potentially able to produce both heat transfer and mixing: a pair of delta winglets and a pair of rectangular winglets. These two types of turbulence promoters integrated in a heat exchanger have flexible design and high heat-transfer performance, and are potentially suitable for chemical reaction.

The aim of this paper is to provide specific comparisons to evaluate the accuracy of advanced numerical methods for flow and heat-transfer predictions inside MHE reactors with vortex generators where experimental data are available. Because of the complex flow structure in such geometries, their performance is generally determined experimentally. This paper first reviews their thermalhydraulic performance, based on data from the literature and computational fluid dynamics (CFD) analysis. Then the mixing capacity of these turbulence promoters is determined using CFD methods. The pertinent choice of turbulence models according to the geometry is examined and discussed, and the macro- and micro-mixing ability of the vortex generators is addressed.

\section{Vortex generators}

Inside a duct channel, vortices can be generated by flow separation that makes the fluid rotate continuously around their rotation axis. This phenomenon can be used to enhance convective heat transfer.

\subsection{Design and physical phenomena}

Most of the vortex generators (VGs) designed on the above concepts have been manufactured by punching or embossing the wall of a plate or a channel. The present study focuses only on the punched geometry. The threedimensional nature of VG allows a large variety of configurations. Two different basic vortex generator forms are investigated here: delta forms and rectangular forms. Wing and winglet VGs, mounted and punched VGs, single rows, double rows and periodic arrays of VGs were investigated. This paper focuses on rectangular winglet pairs (RWP) (Fig. 1) and delta winglet pairs (DWP) (Fig. 2).

Vortex generators, while remaining attached to the plate at the base, protrude into the flow at an angle of attack to the main flow direction. The two most important dimensionless geometric parameters that control vortex characteristics (e.g. heat transfer and pressure loss enhancement) are this angle of attack and the VG area.

The basic principle of vortex generators is based on the cutoff of the thermal boundary layer developed along the wall and also on the heat removal from the wall to the core of the flow by means of large-scale turbulence. An efficient way to enhance heat transfer is to generate counter-rotating vortices by means of pairs of delta or rectangular wing-

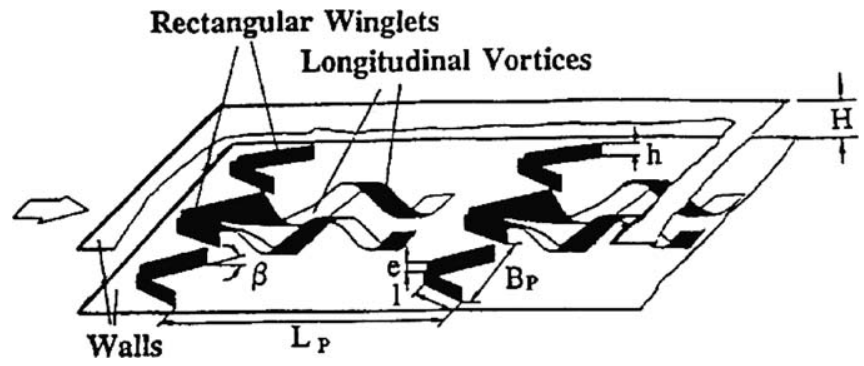

Fig. 1. Rectangular vortex generator geometry and notation.

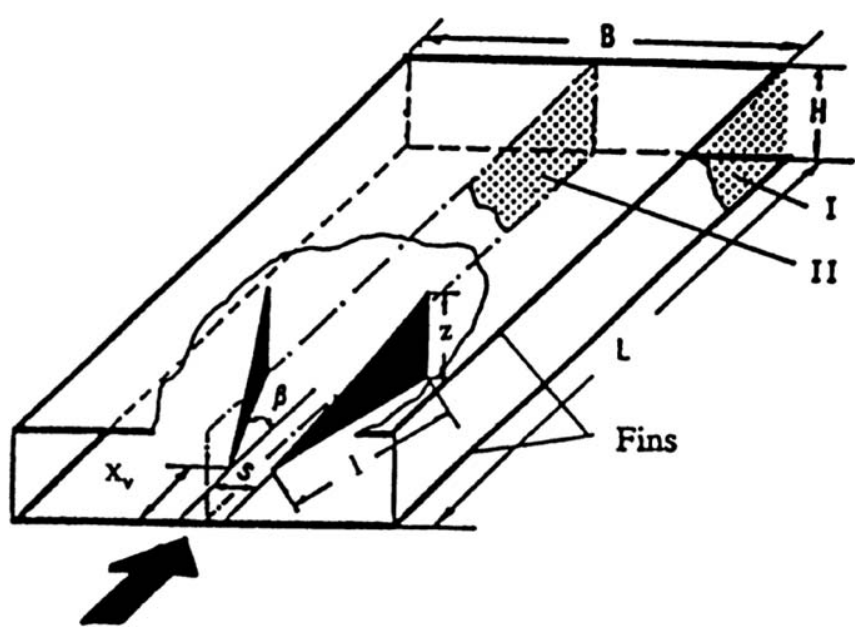

Fig. 2. Triangular vortex generator geometry and notation.

lets. Single elements have been shown to have lower performance $[11,12]$. With VGs, the transition to turbulence occurs at lower Reynolds numbers than in a plane channel flow and the turbulence intensity is increased $[13,14]$. Vortex generators seem to be appropriate heattransfer enhancing devices in flows with intermediate Reynolds numbers. Delta forms are slightly more efficient than rectangular forms [12].

Transverse vortices may be distinguished from longitudinal ones. The predominance of one over the other depends on the angle of attack $\beta[9,11]$. Transverse vortices have their axes perpendicular to the flow direction and are consistent with two-dimensional flow. Longitudinal vortices have their axes in the streamwise or flow direction and always lead to a three-dimensional flow. For $\beta \leqslant 65^{\circ}$ the vortex system is dominated by longitudinal vortices, while for $\beta \geqslant 70^{\circ}$ transverse vortex structures are dominant. For RWP and DWP, it has been shown that longitudinal vortices are more efficient than transverse ones when pressure losses are taken into account $[9,15]$. Heat transfer does not increase substantially for angles of attack larger than $65^{\circ}$.

\subsection{Overall performance of vortex generators}

Pairs of winglets (triangular or rectangular) have been studied experimentally and numerically by Tiggelbeck 
Table 1

Performance of VGs at $R e=4600$ and $\beta=30^{\circ}$ [12]

\begin{tabular}{llllll}
\hline Geometry & $N u$ & $N u / N u_{0}$ & $f / f_{0}$ & $j$ & $j / f$ \\
\hline Triangular & 24.76 & 1.49 & 1.91 & 0.0061 & 0.39 \\
Rectangular & 24.26 & 1.46 & 1.85 & 0.0059 & 0.39 \\
\hline
\end{tabular}

et al. [12]. The triangular winglet height was equal to the channel height while the rectangular winglet height was only half the channel height. Available experimental data, in terms of Nusselt number, friction factor and Colburn factor, are summarized in Table 1.

The Colburn factor is defined by

$j=\frac{N u}{\operatorname{RePr}{ }^{1 / 3}}$

where the Reynolds number $R e$ is given by

$R e=\frac{\rho U d_{\mathrm{h}}}{\mu}$

The Fanning friction factor is defined by

$f=\frac{\Delta P / L}{2 d_{\mathrm{h}} \rho U^{2}}$

In Table 1, subscript 0 represents the reference state which is empty duct channel (without inserts). These results show VG abilities to enhance heat transfer without a dramatic increase of the pressure drops compared with compact plate-fin heat exchangers which generate very high heattransfer enhancement with unfortunately impressive pressure drops. Thus, the $j / f$ ratio for vortex generators is better $(j / f>0.39)$ than for classical compact plate-fin heat exchanger. Moreover, within the range of Reynolds numbers tested (2000-8000), the heat transfer and pressure drop enhancement are proportional to $R e^{0.3}$.

\section{Numerical models and procedure}

A numerical simulation of air flow inside a duct with one pair of winglets was performed using FLUENT software with different turbulence models.

\subsection{Turbulence models}

Several turbulence models were used in this study. The $(k-\varepsilon)$ model and the large eddy simulation (LES) were tested with different model laws: standard [16], RNG [17] and Realizable [18] for the $(k-\varepsilon)$ model and Smagorinsky $[19,20]$ and RNG $[21]$ for the LES model.

The standard $(k-\varepsilon)$ model [16] is a semi-empirical model based on model transport equations for the turbulence kinetic energy $(k)$ and its dissipation rate $(\varepsilon)$. The model transport equation for $k$ was derived from the exact equations, while the model transport equation for $\varepsilon$ was obtained by phenomenological reasoning and bears little resemblance to its mathematically exact counterpart.

In deriving the $(k-\varepsilon)$ model, it was assumed that the flow is fully turbulent and the effects of molecular viscosity are negligible. The standard $(k-\varepsilon)$ model is therefore valid only for fully turbulent flows. The RNG-based $(k-\varepsilon)$ turbulence model [17] is derived from the instantaneous Navier-Stokes equations by using a mathematical technique called the "renormalization group" (RNG) method. The analytical derivation yields a model with constants different from those in the standard $(k-\varepsilon)$ model, and additional terms and functions appear in the transport equations for $(k-\varepsilon)$.

In addition to the standard and RNG-based $(k-\varepsilon)$ models described above, FLUENT software also provides the so-called Realizable $(k-\varepsilon)$ model [18]. The term "Realizable" means that the model satisfies certain mathematical constraints on the normal stresses that are consistent with the physics of turbulent flows.

Unsteady simulations were carried out using a Smagorinsky [19,20] and RNG [21] LES turbulence models. In these simulations, transient conservation equations for mass, momentum and energy were solved until an equilibrium turbulence is achieved. Therefore, these results are time averaged. Time step has been fixed to a constant value, $10^{-5} \mathrm{~s}$, which is in agreement with the C.F.L. (Courant-Friedrichs-Levy) conditions. The main results of these simulations are presented below.

\subsection{Mixing model}

Knowledge of the rate of turbulence energy dissipation, $\varepsilon$, is needed to predict the micro-mixing ability of a device. The average energy dissipation rate, $\Phi$, is related to the volumetric flow rate $Q$, the pressure drop $\Delta P$, the fluid density $\rho$ and the working volume $V_{\mathrm{M}}$ by

$\Phi=\frac{Q \Delta P}{V_{\mathrm{M}} \rho}$

$\Phi$ is expressed per unit mass of fluid in the channel, so that $V_{\mathrm{M}}$ is the total internal volume less the volume of the metal internals. The working volume is the total internal volume multiplied by the fractional liquid hold-up $e$. The dissipation rate $\Phi$ can be divided into turbulent dissipation $\varepsilon$, caused by gradients in the turbulent velocity fluctuations, and direct dissipation $E_{\mathrm{D}}$, due to gradients of the mean velocity. Thus:

$\Phi=\varepsilon+E_{\mathrm{D}}$

where $\Phi$ is averaged over the volume $V_{\mathrm{M}} \varepsilon$ and $E_{\mathrm{D}}$ generally vary widely with position in the channel.

The pressure drop is related to the friction factor by

$\Delta P=4 f \frac{Q^{2}}{2 \rho} \frac{L}{d_{\mathrm{h}}}$

$f$ is defined as before.

The mixing efficiency characterizes the ratio of the rate of turbulent kinetic energy dissipation to the total (averaged) energy dissipation rate. This mixing efficiency can be determined by the value of the pressure drop and the turbulence energy dissipation $\varepsilon\left(\mathrm{m}^{2} \mathrm{~s}^{-3}\right)$ : 
$\eta=\frac{\varepsilon}{\Phi}=1-\frac{E_{\mathrm{D}}}{\Phi}$

For the numerical computations, the above method can be used to evaluate the mixing efficiency. Thus one must estimate the pressure drop $\Delta P$ and rate of the turbulence energy dissipation $\varepsilon$ by using a $(k-\varepsilon)$ or a LES model. Then the pressure drop calculation lets one compute the average energy dissipation rate, $\Phi$, from which the mixing efficiency can be deduced.

\subsection{Geometry and grid}

The main dimensions of the two geometries are given in Table 2. The geometries are meshed with tetrahedral elements for the delta winglet pair and hexagonal elements for the rectangular winglet pair. The effect of grid resolution on the results has been tested on rectangular winglet pair at Reynolds number 4600 and angle of attack $30^{\circ}$. Three different meshes have been studied with a $(k-\varepsilon)$ RNG model as showed in Table 3.

These results seems to be invariant for mesh element numbers higher than 120000. Consequently, in order to reduce computation time, the overall numbers of elements are chosen around 120000 for the RWP and by keeping the same interval size 250000 for the DWP.

Table 2

Geometrical dimensions of the winglets

\begin{tabular}{|c|c|c|c|c|}
\hline & Tiggelbeck [11] & $\operatorname{RWP}\left(30^{\circ}\right)$ & $\operatorname{RWP}\left(65^{\circ}\right)$ & $\operatorname{DWP}\left(65^{\circ}\right)$ \\
\hline $\begin{array}{l}\text { Channel } \\
\text { height } H\end{array}$ & $20 \mathrm{~mm}$ & $6.35 \mathrm{~mm}$ & $6.35 \mathrm{~mm}$ & $5 \mathrm{~mm}$ \\
\hline $\begin{array}{l}\text { Channel } \\
\text { length } L\end{array}$ & $15 H$ & $15 H$ & $15 H$ & $15 H$ \\
\hline $\begin{array}{l}\text { Channel } \\
\quad \text { breadth } B\end{array}$ & $5 H$ & $5 H$ & $5 H$ & $5 H$ \\
\hline Fin area $A_{\mathrm{F}}$ & $30000 \mathrm{~mm}^{2}$ & $3024 \mathrm{~mm}^{2}$ & $3024 \mathrm{~mm}^{2}$ & $1875 \mathrm{~mm}^{2}$ \\
\hline $\begin{array}{l}\text { Angle of } \\
\quad \text { attack } \beta\end{array}$ & $65^{\circ}$ or $30^{\circ}$ & $30^{\circ}$ & $65^{\circ}$ & $65^{\circ}$ \\
\hline $\begin{array}{l}\text { Height of } \\
\text { VG } z\end{array}$ & $H$ & $H$ & $H$ & $H$ \\
\hline $\begin{array}{c}\text { Span of } \\
\text { VG } l\end{array}$ & $2 H$ & $2 H$ & $2 H$ & $2 H$ \\
\hline $\begin{array}{l}\text { Area of } \\
\quad \text { VG } A_{\mathrm{VG}}\end{array}$ & $800 \mathrm{~mm}^{2}$ & $80 \mathrm{~mm}^{2}$ & $80 \mathrm{~mm}^{2}$ & $50 \mathrm{~mm}^{2}$ \\
\hline$A_{\mathrm{F}} / A_{\mathrm{VG}}$ & 37.5 & 37.5 & 37.5 & 37.5 \\
\hline$x_{v}$ & $H$ & $H$ & $H$ & $H$ \\
\hline$s$ & $0.2 H$ & $0.2 H$ & $0.2 H$ & $0.2 H$ \\
\hline
\end{tabular}

Table 3

Influence of the mesh on simulation results for a RWP at $R e=4600$ and $\beta=30^{\circ}$

\begin{tabular}{lll}
\hline Mesh elements number & $N u / N u_{0}$ & $C f / C f_{0}$ \\
\hline 40000 & 1.4 & 1.7 \\
120000 & 1.4 & 1.8 \\
220000 & 1.4 & 1.8 \\
\hline
\end{tabular}

\section{Numerical results}

\subsection{Results for $R W P$ at $R e=4600$ and $30^{\circ}$ angle of attack}

Several turbulence models were tested for a rectangular winglet pair at Reynolds number 4600 and angle of attack $30^{\circ}$. The main dimensions of this geometry are shown in Table 2. The $(k-\varepsilon)$ and LES models were tested with different model laws: standard, RNG and Realizable for the $(k-\varepsilon)$ model and Smagorinsky and RNG for the LES model. Furthermore, for the LES turbulent model, unsteady simulations were carried out. These results have been obtained by using Gnielinski correlation [22] and are summarized in Table 4 . In this table, subscript 0 indicates the reference state which is empty duct channel (without inserts).

Comparing the above numerical results with experimental data from [12], it can be seen that the $(k-\varepsilon)$ and the LES models satisfactorily predict heat-transfer enhancement $\left(\mathrm{Nu} / \mathrm{Nu} u_{0}\right)$ and drag coefficient enhancement $\left(C f / C f_{0}\right)$.

The RNG and Realizable $(k-\varepsilon)$ turbulent models seem to give the best results. Indeed, they have specific terms to take into account swirl and adverse pressure gradients. A comparison of the LES computations leads to the conclusion that the generation of longitudinal vortices is actually a quasi-steady phenomenon. Indeed, there is no significant difference between these two simulations in terms of heat transfer and drag enhancement. As expected, both RNG or Realizable $(k-\varepsilon)$ and LES simulations describe a pair of counter-rotating vortices in the wake of the winglets, but the standard $(k-\varepsilon)$ model does not correctly predict counter-rotating vortices (Fig. 3). These vortices are generated by the pressure difference between the pressure side and suction side of the wings.

\subsection{Effects of the angle of attack for $R W P$}

The influence of the angle of attack $\beta$ was also studied with the best turbulence models. Using a LES model with a RNG subgrid scale model and a RNG $(k-\varepsilon)$ model, computations on the rectangular winglet pair were carried out for Reynolds number 4600 and angle of attack $65^{\circ}$. These results are also compared with those obtained previously for $30^{\circ}$ (Table 5).

Table 4

Results for a RWP at $R e=4600$ and $\beta=30^{\circ}$

\begin{tabular}{lrrrrll}
\hline & {$[12]$} & $\begin{array}{l}k-\varepsilon \\
\text { Std }\end{array}$ & $\begin{array}{l}k-\varepsilon \\
\text { RNG }\end{array}$ & $\begin{array}{l}k-\varepsilon \\
\text { Rlz }\end{array}$ & $\begin{array}{l}\text { LES } \\
\text { Smago }\end{array}$ & $\begin{array}{l}\text { LES } \\
\text { RNG }\end{array}$ \\
\hline$N u$ & 24.3 & 30.7 & 27.2 & 29.5 & 32.0 & 31.5 \\
$N u_{0}$ & 16.6 & 19.3 & 19.3 & 19.3 & 19.3 & 19.3 \\
$N u / N u_{0}$ & 1.5 & 1.6 & 1.4 & 1.5 & 1.7 & 1.6 \\
$C f\left(\times 10^{2}\right)$ & 2.8 & 3.3 & 2.9 & 3.0 & 3.4 & 3.4 \\
$C f_{0}\left(\times 10^{2}\right)$ & 1.5 & 1.7 & 1.7 & 1.7 & 2.0 & 2.0 \\
$C f / C f_{0}$ & 1.9 & 1.9 & 1.8 & 1.8 & 1.7 & 1.7 \\
\hline
\end{tabular}




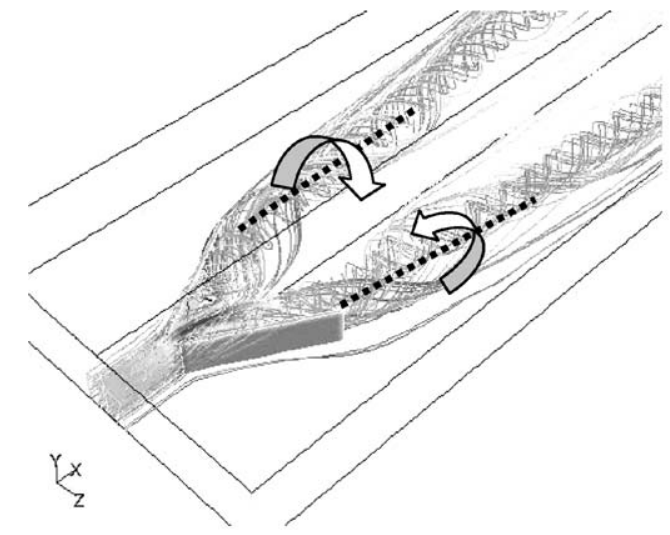

(a)

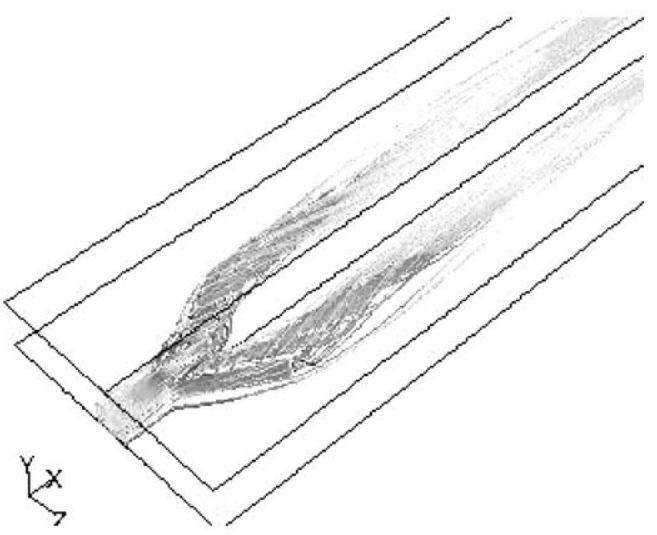

(b)

Fig. 3. Streamlines in the wake of a RWP $\left(30^{\circ}\right)$ with LES model (a) and with $(k-\varepsilon)$ standard model (b).

Table 5

Effect of angle of attack on Nusselt enhancement and drag coefficient for a $\mathrm{RWP}$ at $R e=4600$

\begin{tabular}{|c|c|c|c|c|c|c|}
\hline & \multicolumn{3}{|l|}{$30^{\circ}$} & \multicolumn{3}{|l|}{$65^{\circ}$} \\
\hline & [12] & $(k-\varepsilon)$ & LES & [12] & $(k-\varepsilon)$ & LES \\
\hline$N u / N u_{0}$ & 1.5 & 1.5 & 1.6 & 1.7 & 1.8 & 1.8 \\
\hline$C f / C f_{0}$ & 1.9 & 1.7 & 1.8 & 3.3 & 3.8 & 3.4 \\
\hline
\end{tabular}

Comparing the above numerical results with experimental data from [12] shows that the LES simulation gives good predictions in term of heat transfer and pressure losses for both angles. The RNG $(k-\varepsilon)$ model strongly overestimates the pressure drop for $65^{\circ}$ angle. Indeed, a lit- erature survey shows that for high angles of attack, the flow behaviour in the wake of a VG moves from longitudinal to transverse vortices. Thus $(k-\varepsilon)$ models, even with swirl and pressure gradient effect, can no longer predict that kind of strong anisotropic flow patterns. Therefore, the LES model is the only one able to describe qualitatively and quantitatively the flow behaviour for various angles.

For both results, heat transfer and drag coefficient increase with angle of attack. Moreover, the LES computation shows the transition domain between transversal and longitudinal vortices for a $65^{\circ}$ angle of attack [9]. Indeed, contrary to Fig. 3, which shows a predominance of longitudinal vortices for $30^{\circ}$ angle of attack, Fig. 4 shows that both longitudinal and transversal vortices exist.

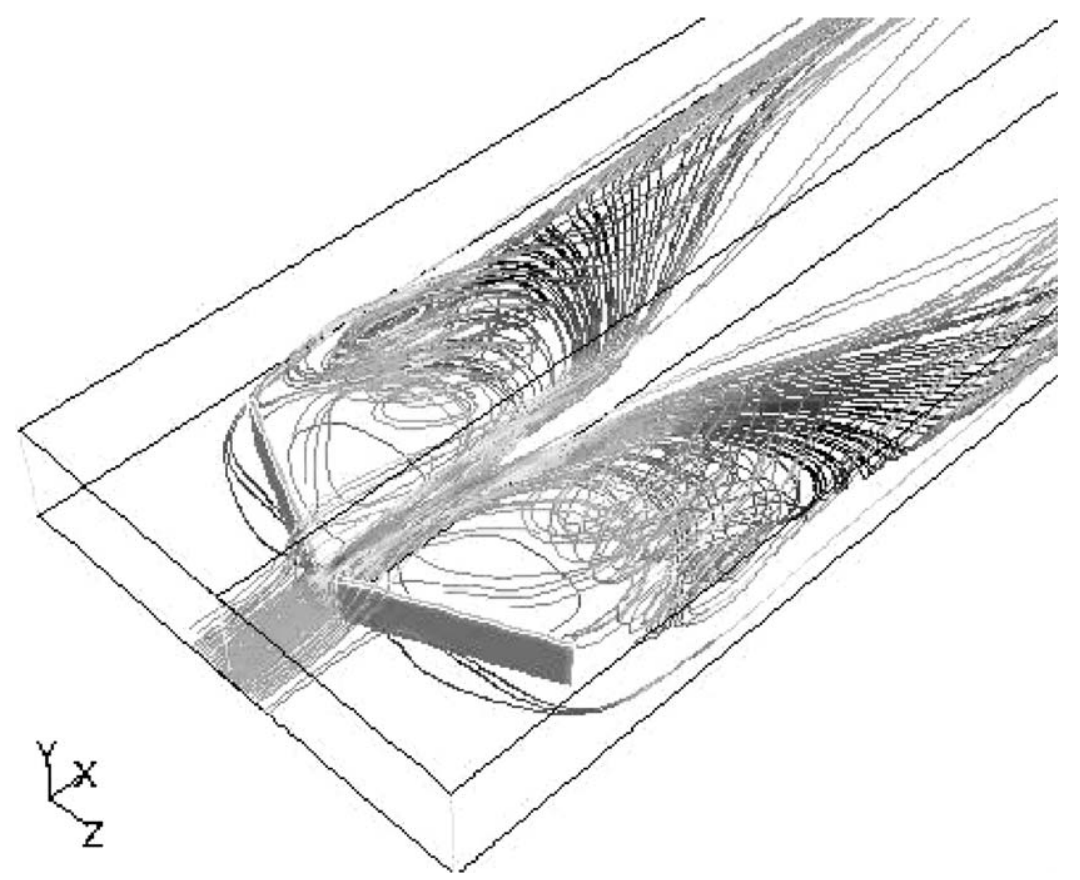

Fig. 4. Streamlines in the wake of a RWP $\left(65^{\circ}\right)$ with LES model. 


\subsection{Effects of Reynolds number for RWP}

According to the results obtained for RWP at two different angles of attack, the LES turbulence model is the only one able to produce satisfactory information for mainly longitudinal vortices $\left(30^{\circ}\right)$ or mainly transverse ones $\left(65^{\circ}\right)$. Therefore, this model was used to study the effects of Reynolds number (2000-8000) at an angle of attack of $65^{\circ}$. The results are then compared with the available data in Figs. 5 and 6.

LES computations show satisfactory prediction of the drag coefficient $\left(C f / C f_{0}\right)$ for Reynolds numbers lower than 6000 (Fig. 5). However, for Reynolds numbers higher than 6000 , a difference arises between experimental data from [12] and LES computations. This difference may be due to the coarseness of the mesh at this Reynolds number, where the boundary layer is thicker. Thus, for Reynolds numbers higher than 6000 , it may be useful to increase the mesh element numbers. The heat-transfer prediction seems to be satisfactory with a LES computation for the entire turbulent flow regime. Nevertheless, this simulation is not satisfactory for Reynolds numbers around 2000, i.e. for a laminar flow regime $\left(N u / N u_{0}=1.9\right.$ instead of 1.4); therefore, LES computations overestimate the heat transfer for Reynolds numbers below 2300. Consequently,

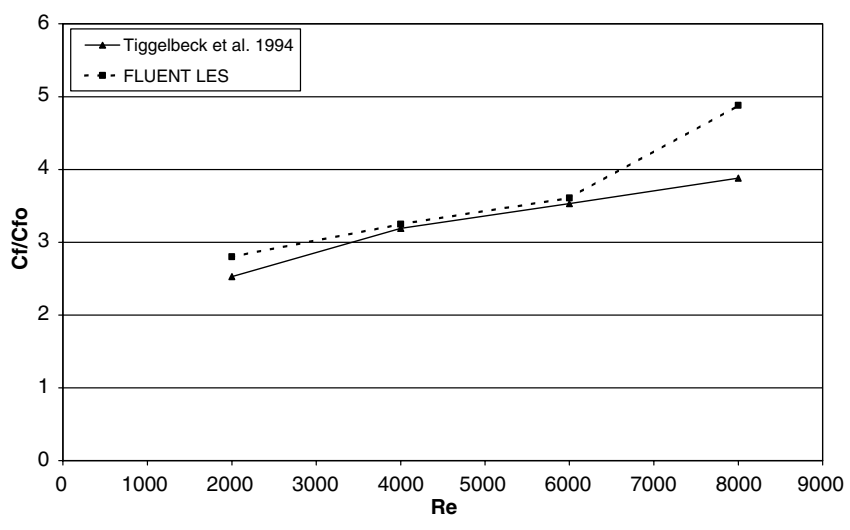

Fig. 5. Drag coefficient vs. Reynolds number for RWP.

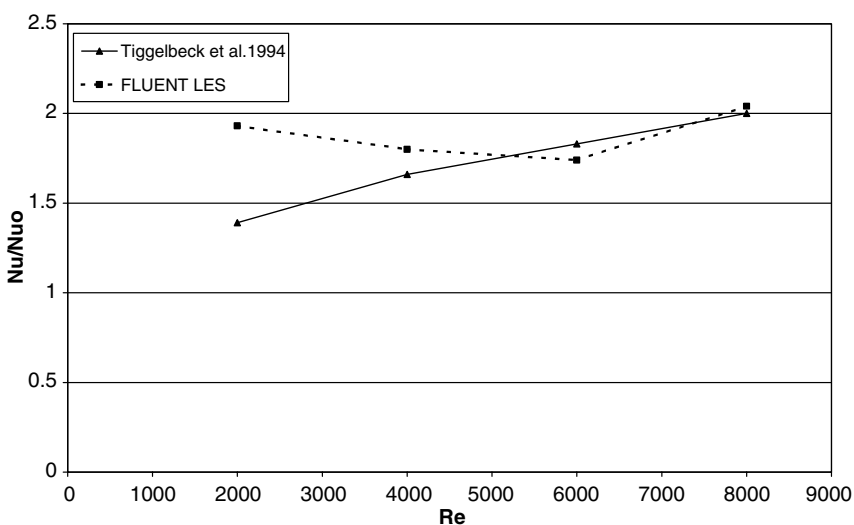

Fig. 6. Nusselt number enhancement vs. Reynolds number for RWP. a laminar model has been used for Reynolds number of 2000. This computation gives $N u / N u_{0}=1.4$, the same as the experimental value in Fig. 6 , but $C f / C f_{0}=3.1$ instead of 2.8 with LES model. Thus, laminar flow simulation gives globally appropriate results for Reynolds numbers lower than 2300. Moreover, Fig. 6 shows a minimum Nusselt number enhancement for Reynolds number equal to 6000. This minimum has no physical significance, it should reflect the difficulties of numerical simulations in the transitional region of the flow.

\subsection{Effects of Reynolds number for DWP}

Using the LES turbulence model with a RNG subgrid scale model, simulations for the delta winglet pair were carried out for different Reynolds numbers (2000-8000) and a $65^{\circ}$ angle of attack. This geometry leads to the formation of a pair of counter-rotating vortices in the wake of the winglets as is shown in Fig. 7.

Comparing the LES results with experimental data from [12], one can readily observe the satisfactory predictions of drag coefficient $\left(C f / C f_{0}\right)$ and heat transfer $\left(N u / N u_{0}\right)$ by these computations (Figs. 8 and 9) over the entire Reynolds number range.

The drag coefficient in DWP configuration is of the same order of magnitude as in RWP. Indeed, the friction enhancement varies in the two cases from 2.7 to about 4 . A small heat-transfer enhancement difference was observed between DWP and RWP (1.5 instead of 1.4 at $R e=2000$; 2.2 instead of 2.0 at $R e=8000$ ).

\subsection{Comparison of the compactness of $R W P$ and $D W P$}

In order to compare RWP and DWP performances, the volume minimization criterion $\left(N u / N u_{0}\right)^{3} /\left(C f / C f_{0}\right)$ has been studied based on Tiggelbeck's results [12]. This criterion takes into account heat transfer enhancement against drag increase and allows to compare different geometries in order to increase multifunctional heat exchanger compactness. Fig. 10 shows that DWP allows a better compactness than RWP. Moreover, the difference between DWP and RWP volume minimization criterion increases with Reynolds number. For high Reynolds numbers RWP pressure drop becomes a main drawback and thus DWP seems to be more efficient in terms of compactness.

\section{Mixing performance}

Mixing performance was studied for RWP at $R e=4600$ and $\beta=30^{\circ}$ with a RNG $(k-\varepsilon)$ turbulence model. The $(k-\varepsilon)$ computation allows plotting mixing efficiency vs. $x / H$ (downstream coordinate) as is shown in Fig. 11.

The rectangular winglet pair significantly increases the turbulence energy dissipation rate and thus mixing efficiency, which is highest in the vicinity of the VG and decreases downstream. The high mixing efficiency at the outlet of the channel is due to the large local turbulence 


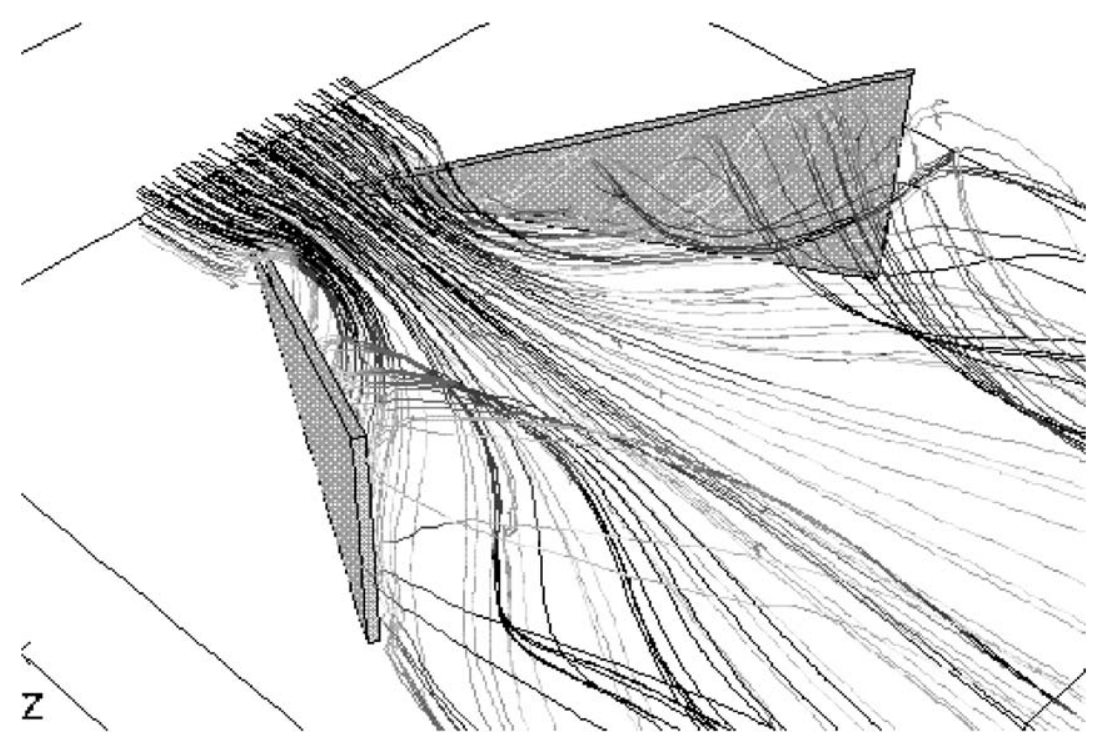

Fig. 7. Streamlines in the wake of a DWP with LES model at $R e=4600$ and $\beta=65^{\circ}$.

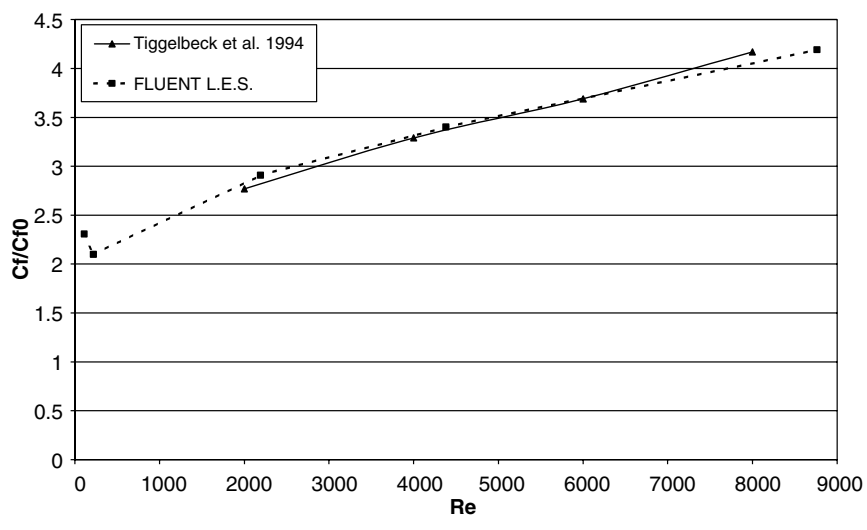

Fig. 8. Drag coefficient vs. Reynolds number for DWP.

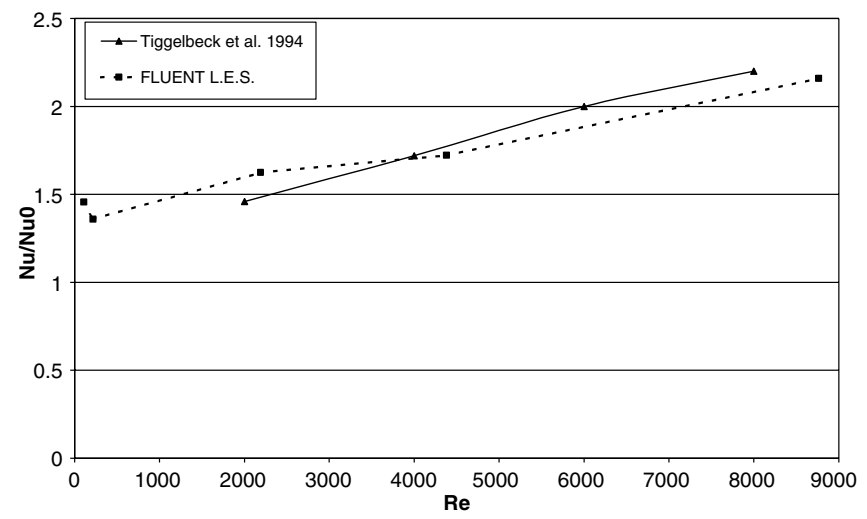

Fig. 9. Nusselt number enhancement vs. Reynolds number for DWP.

energy dissipation rate and thus the large inhomogeneity in the channel generated by longitudinal vortices. Indeed, vorticity along the flow direction reaches a high level $\left(5000 \mathrm{~s}^{-1}\right)$ at the core of the longitudinal vortices (Fig. 12). In other words, the circumferential velocity value

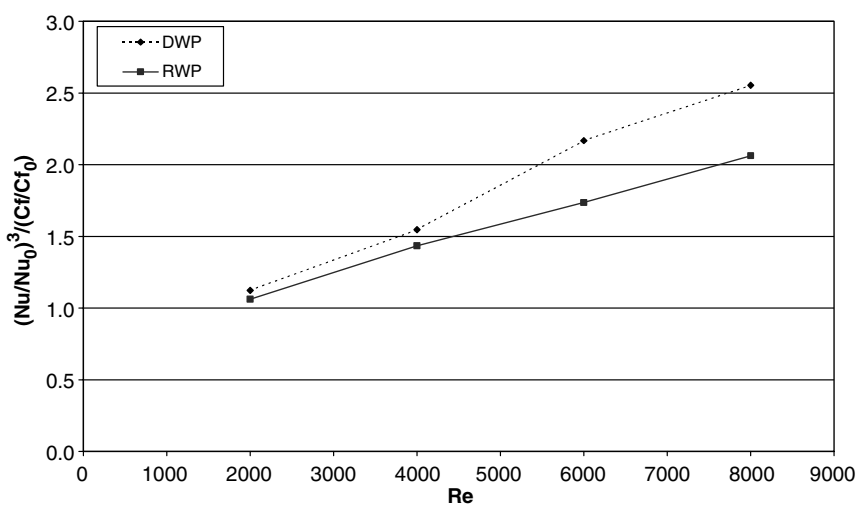

Fig. 10. RWP and DWP compactness comparison at $\beta=65^{\circ}$.

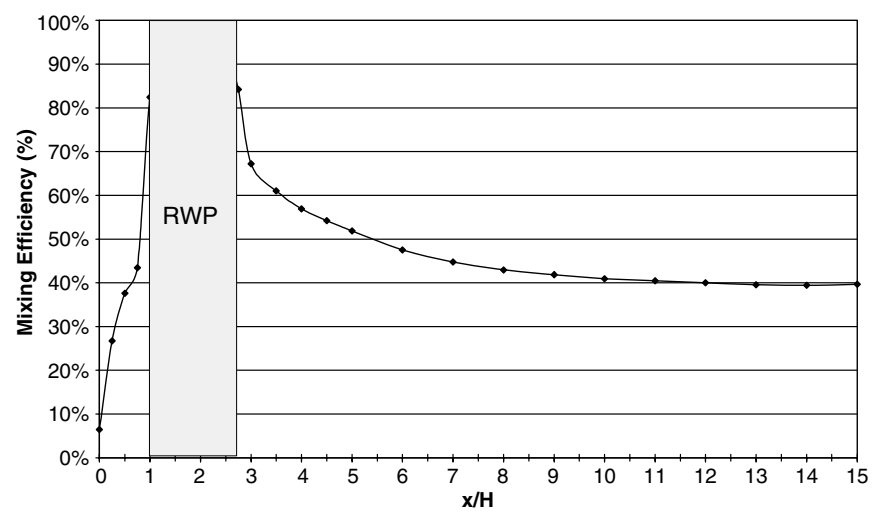

Fig. 11. Mixing efficiency vs. $x / H$ for a RWP at $R e=4600$ and $\beta=30^{\circ}$.

is of the same order of magnitude as the mean axial velocity. This large circumferential velocity implies an increase of shear stress on the wall. These high values of vorticity and circumferential velocity imply a high turbulence energy dissipation rate and thus high mixing efficiency. These results are in agreement with Tiggelbeck et al. results [13] 


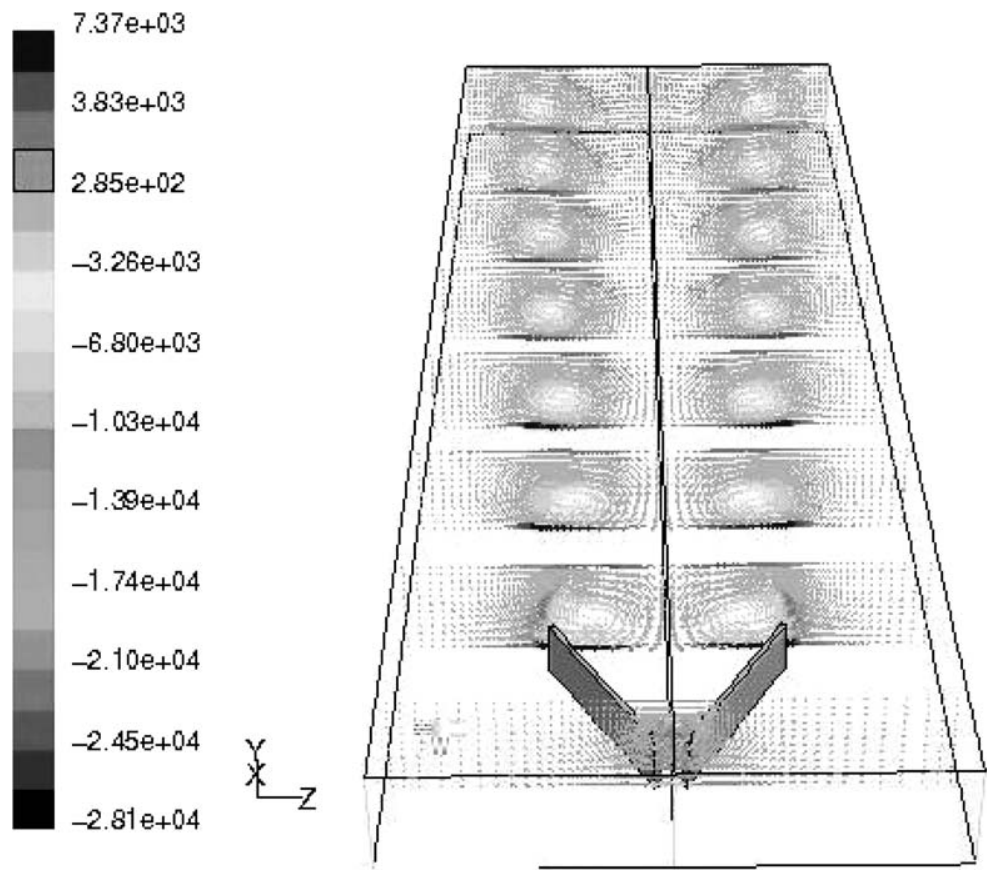

Fig. 12. Streamwise vorticity for various cross-sections along the channel for RWP at $R e=4600$ and $\beta=30^{\circ}$.

which show that a distance $7-10$ times the channel height between vortex generator rows gives highest local and average heat transfer. Indeed, Fig. 11 shows that mixing efficiency falls downstream of the VGs and becomes minimum for a distance 7-10 times the channel height downstream of the VG. This observation confirms that heat and mass transfer depend on hydrodynamic flow.
Temperature iso-contour lines for several cross-sections along the channel shown in Fig. 13 reveal the macro-mixing generated by RWP. These vortices turn the flow field perpendicular to the main flow direction and enhance mixing between the fluid close to the fin and that in the middle of the channel. This mixing causes a local heat-transfer enhancement by the cutoff of the thermal boundary layer
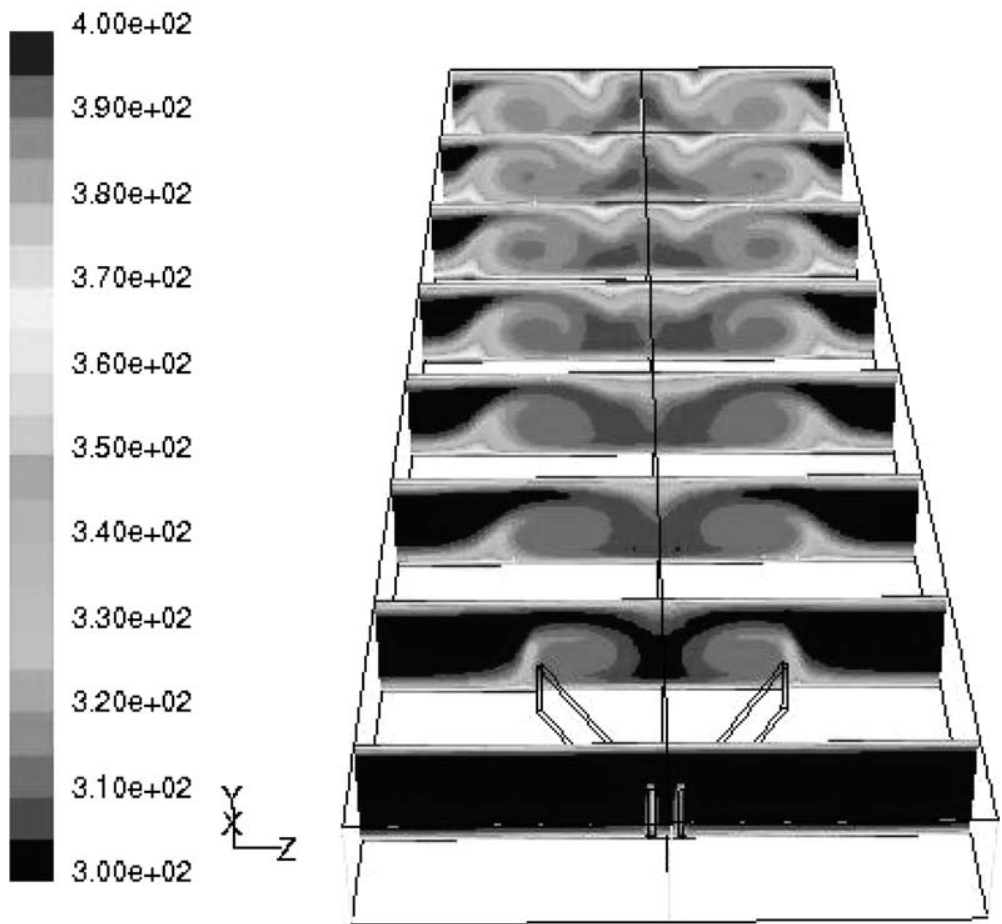

Fig. 13. Temperature contours for various cross-sections along the channel for RWP at $R e=4600$ and $\beta=30^{\circ}$. 
developed along the wall and also the heat transfer from the wall to the core of the flow.

\section{Concluding remarks}

Numerical simulations of vortex generators with advanced turbulence models and refined mesh give reliable qualitative and quantitative results. The present study improves our knowledge of turbulent flow, heat transfer and the mixing ability of compact heat-exchanger geometries. By using steady RANS and unsteady large eddy simulation turbulence models, time averaged results were obtained. For the two vortex generators considered here, the computations with a refined mesh give satisfactory results: the underlying physical phenomena are described and the main geometrical parameters and their effect on turbulence are identified. This study shows that DWP is more efficient than RWP in terms of compactness criterion. Heat transfer and mixing efficiencies are optimum when distance between VG rows are around 7-10 times the channel heights.

For heat-exchanger applications, unsteady computation with a large number of time steps provides no extra information, since the vortices generated are almost steady.

Different numerical models were tested and LES models were validated for a wide range of Reynolds numbers and angles of attack. Thus this methodology is now available for geometry optimisations such as the lateral and longitudinal winglet placement to provide a given turbulence energy dissipation rate in the winglets' wake. This work has shown the utility of advanced numerical methods for optimisation of compact heat-exchanger geometries; they are now available as a tool for design engineers.

\section{Acknowledgements}

S. Ferrouillat acknowledges the financial support of ADEME (French Agency for Energy and Environment).

\section{References}

[1] S. Ferrouillat, P. Tochon, H. Peerhossaini, D. Della Valle, Open-loop thermal control of exothermal chemical reactions in multifunctional heat exchangers, Int. J. Heat Mass Transfer, in press.

[2] S. Ferrouillat, P. Tochon, H. Peerhossaini, Micromixing enhancement by turbulence: application to multifunctional heat exchangers, Chem. Eng. Process., in press.
[3] S. Hardt, W. Ehrfeld, V. Hessel, K.M. Vanden Bussche, Strategies for size reduction of microreactors by heat transfer enhancement effects, Chem. Eng. Commun. 190 (4) (2003) 540-559.

[4] N. Mazet, H.B. Lu, Improving the performance of the reactor under unfavourable operating conditions of low pressure, Appl. Therm. Eng. 18 (9-10) (1998) 819-835.

[5] H. Peerhossaini, On the effects of streamwise vortices on wall heat transfer, in: R. Shah (Ed.), Compact Heat Exchangers for Process Industries, Begell House, 1997, pp. 571-589.

[6] H. Peerhossaini, J.E. Wesfreid, On the inner structure of streamwise Görtler rolls, Int. J. Heat Fluid Flow 9 (1988) 12-18.

[7] L. Momayez, P. Dupont, H. Peerhossaini, Some unexpected effects of wavelength and perturbation strength on heat transfer enhancement by Görtler vortices, Int. J. Heat Mass Transfer 47 (2004) 3783-3795.

[8] L. Momayez, P. Dupont, H. Peerhossaini, Effects of vortex organization on heat transfer enhancement by Görtler vortices, Int. J. Therm. Sci. 43 (2004) 753-760.

[9] M. Fiebig, Vortex generators for compact heat Exchangers, J. Enhanced Heat Transfer 2 (1-2) (1995) 43-61.

[10] D. Eiebeck, J.K. Eaton, Heat transfer effects of longitudinal vortex embedded in a boundary layer, ASME J. Heat Transfer 109 (1987) $16-24$.

[11] M. Fiebig, T. Güntermann, A class of high performance compact finplate heat exchanger elements, Transport Phenomena in Thermal Engineering, Begell House, 1993, pp. 926-931.

[12] S. Tiggelbeck, N.K. Mitra, M. Fiebig, Comparison of wing-type vortex generators for heat transfer enhancement in channel flows, ASME J. Heat Transfer 116 (1994) 880-885.

[13] S. Tiggelbeck, N.K. Mitra, M. Fiebig, Experimental investigations of heat transfer and flow losses in a channel with double rows if longitudinal vortex generators, Int. J. Heat Mass Transfer 36 (1993) 2327-2337.

[14] M. Fiebig, P. Kallweit, N.K. Mitra, Wing type vortex generators for heat transfer enhancement, I.H.T.C. 6 (1986) 2909-2913.

[15] J.I. Yanagihara, K. Torii, Heat transfer characteristics of laminar boundary layer in the presence of vortex generators, in: Proc. 9th IHTC, vol. 6, 1990, pp. 323-328.

[16] B.E. Launder, D.B. Spalding, Lectures in Mathematical Models of Turbulence, Academic Press, London, England, 1972.

[17] V. Yakhot, S.A. Orszag, Renormalization Group Analysis of Turbulence: I. Basic Theory, J. Sci. Comput. 1 (1986) 1-51.

[18] T.H. Shih, W.W. Liou, A. Shabbir, J. Zhu, A new $k-\varepsilon$ eddy-viscosity model for high Reynolds number turbulent flows-model development and validation, Comput. Fluids 24 (1995) 227-238.

[19] U. Schumann, Subgrid scale model for finite difference simulations of turbulent flows in plane channels and annuli, J. Comput. Phys. 18 (1975) 376-404.

[20] J.S. Smagorinsky, General circulation experiments with the primitive equations: I — The basic experiments, Mon. Weather Rev. 91 (1963) 99-164.

[21] A. Yakhot, S.A. Orszag, V. Yakhot, M. Israeli, Renormalization group formulation of large-eddy simulation, J. Sci. Comput. 4 (1989) $139-158$.

[22] V. Gnielinski, New equation for heat and mass transfer in turbulent pipe and channel flow, Int. Chem. Eng. 16 (1976) 359-368. 